\title{
Osteocalcin, osteopontin and RUNX2 expression in patients with arteriosclerosis
}

\author{
JÖRG UKKAT, CUONG HOANG-VU, BOGUSZ TROJANOWICZ and ARTUR REBELO \\ Department of Visceral, Vascular and Endocrine Surgery, Martin Luther \\ University Halle-Wittenberg, 06120 Halle (Saale), Germany
}

Received February 10, 2021; Accepted April 20, 2021

DOI: $10.3892 /$ wasj.2021.103

\begin{abstract}
Knowledge of the role of the skeleton as an endocrine organ has increase over the past years. The role of calcification in arteriosclerotic disease has also been a matter of investigation. Since there is a clear association between the levels of osteocalcin (OC), osteopontin (OPN) and Runt-related transcription factor 2 (RUNX2) and the development of arteriosclerotic vascular disease, the present study examined the expression of OC, OPN and RUNX2 in vascular tissue and their possible employment as diagnostic markers for cardiovascular disease. For this purpose, 13 vessel tissues, including tissues from six patients without arteriosclerotic disease (PAD-) and 7 patients with arteriosclerotic disease (PAD+) were obtained by surgical resection. Immunohistochemistry and reverse transcription-quantitative polymerase chain reaction (RT-qPCR) analysis were performed. The results revealed that tissues representing PAD+ exhibited a noticeably stronger protein expression of OC, OPN and RUNX2 as compared with the corresponding PAD- tissues. The transcript levels of OPN and RUNX2 were associated with protein expression and were significantly elevated in PAD+ $(\mathrm{OPN}, \mathrm{P}<0.05$; RUNX2, $\mathrm{P}<0.01)$. The expression of $\mathrm{OC}$ in $\mathrm{PAD}+$ was noticeably increased as compared with PAD- $(\mathrm{P}=0.067)$. On the whole, the present study demonstrates that the expression of $\mathrm{OC}$, OPN and RUNX2 in arterial tissue is strongly associated with symptomatic arteriosclerotic disease. Further investigations are required however, to prove the tge clinical significance of OC, OPN and RUNX2 as potential biomarkers for atherosclerotic disease.
\end{abstract}

Correspondence to: Dr Jörg Ukkat or Dr Artur Rebelo, Department of Visceral, Vascular and Endocrine Surgery, Martin Luther University Halle-Wittenberg, Ernst-Grube-Straße 40, 06120 Halle (Saale), Germany

E-mail: joerg.ukkat@uk-halle.de

E-mail: artur.rebelo@uk-halle.de

Key words: osteocalcin, osteopontin, Runt-related transcription factor 2, arteriosclerosis

\section{Introduction}

The understanding of the role of the skeleton as an endocrine organ has increased over the past years (1). Calcification is a highly relevant process in terms of the development of cardiovascular diseases (CVDs). Arteriosclerosis, Mönckeberg medial calcific sclerosis and arteriolosclerosis are the three main lesions derived from classification $(2,3)$. In patients with end-stage chronic kidney disease (CKD), arterial calcification is an independently predictive factor of CVD and mortality (4). The prevention of CVD is currently a highly relevant topic. CVDs are a group of disorders affecting the heart and blood vessels. It has been demonstrated that 17.9 million individuals worldwide succumb to CVDs annually, representing $31 \%$ of all deaths worldwide (5). Thus, establishing novel and reliable predictive biomarkers for the identification of patients with related calcification is a highly relevant topic, of utmost urgency. Vascular calcification is a potential predictor of future cardiovascular events in asymptomatic patients (6).

The collagen-rich matrix that composes bone tissue is produced by osteoblasts. In addition, other regulating functions of these cells have been studied. In the center of this potential hormonal role is osteocalcin (OC). $\mathrm{OC}$ is a bone $\gamma$-carboxyglutamic acid (Gla) protein produced by osteoblasts. Its functions include mineralization, the regulation of glucose and energy metabolism, and the regulation of fertility and cognition (7). One example is the energy metabolism regulation through the adipokine, leptin, acting on osteoblasts and its feedback loop. In a previous study, osteocalcin knockout mouse exhibited glucose intolerance, increased fat mass and insulin resistance (8).

A previous cross-sectional study aimed at evaluating the prevalence of diabetic complications in Chinese patients diagnosed with type 2 diabetes demonstrated that serum OC levels were an independent risk factor for carotid arteriosclerosis in these patients (9).

The role of the OC in the development of atherosclerotic vascular disease remains unclear, however. There is some evidence to indicate that $\mathrm{OC}$ may play a potential role on protecting endothelial function and preventing atherogenesis. On the other hand, OC is associated with vascular calcification, possibly as a mediator or a marker (10). In a previous meta-analysis on this topic, no clear association between OC and vascular calcification or atherosclerosis was found (11). In the studies included in this meta-analysis, the OC concentration 
in blood, the presence of OC-positive cells, or histological staining for $\mathrm{OC}$ were compared to the extent of calcification or atherosclerosis. A total of 26 positive, 17 negative and 29 neutral associations were reported (11).

Osteopontin (OPN) is also a protein with a major role in mineralization and calcification; however, it also has multifunctional roles in physiological and pathophysiological processes. Its role in inflammatory processes has been studied in over the years (12). It has been demonstrated that low levels of OPN may play a protective role concerning vascular tissue injury and high levels may have potentially damaging effects (13). Runt-related transcription factor 2 (RUNX2), is also a bone regulator and has been detected in calcified atherosclerotic lesions $(14,15)$. Furthermore, the role of RUNX2 in vascular calcification has been studied in in recent years $(16,17)$.

Since there is a clear association between OC, OPN and RUNX2, and the development of arteriosclerotic vascular disease, the present study investigated the expression of OC, OPN and RUNX2 in vascular tissue, and their possible employment as diagnostic markers for CVD.

\section{Patients and methods}

Patients and tissue preparation. A total of 13 vessel tissues, including tissues from six patients without arteriosclerotic disease (PAD-) and seven patients with arteriosclerotic disease (PAD+) were collected at the Department of Surgery of Martin-Luther-University Halle, Halle (Saale), Germany by surgical resection (Table I). The patients in the control group (without arteriosclerotic disease) were mostly patients that underwent arterial resections in oncologic surgery. The pathological diagnosis of the tissue sections was confirmed by hematoxylin and eosin (H\&E) staining. All tissues were snap frozen in liquid nitrogen and stored at $-80^{\circ} \mathrm{C}$ for further proceedings. Total RNA was extracted from all samples using TRIzol ${ }^{\circledR}$ reagent (Thermo Fisher Scientific, Inc.) according to the manufacturer's instructions.

Additionally, selected tissues from all groups were investigated in immunohistochemical assays with antibodies specific for OC, OPN and RUNX2, as described below. The Ethics Committee of the Martin Luther University, Faculty of Medicine, approved the study and all patients provided written informed consent.

RNA isolation. Briefly, the vessels were snap-frozen in liquid nitrogen and homogenized in Teflon-Tubes at 2,500 rpm for $45 \mathrm{sec}$ by employing Mikro-Dismembrator S (Braun Biotech International $\mathrm{GmbH}$ ). The resulting powder tissue (ca. $100 \mathrm{mg}$ ) was mixed with $1 \mathrm{ml} \mathrm{TRIzol}{ }^{\circledR}$ reagent, vortexed for $30 \mathrm{sec}$ and incubated at room temperature for $5 \mathrm{~min}$. Following the addition of $0.2 \mathrm{ml}$ chloroform, the samples were centrifuged 15 min by $14,000 \mathrm{x} \mathrm{g}$ at $4^{\circ} \mathrm{C}$ and RNA-containing upper phase was collected and mixed with $0.5 \mathrm{ml}$ isopropanol. Following centrifugation $\left(10 \mathrm{~min}\right.$ by $14,000 \mathrm{xg}$ at $\left.4^{\circ} \mathrm{C}\right)$ the pelleted RNA was washed twice with $75 \%$ ethanol, air-dried and dissolved in RNase-free water. Finally, the dissolved RNA was incubated for $10 \mathrm{~min}$ at $60^{\circ} \mathrm{C}$ and stored at $-80^{\circ} \mathrm{C}$ until use.

Reverse transcription-quantitative polymerase chain reaction $(R T-q P C R)$. A total of $1 \mu \mathrm{g}$ of total RNA was reverse transcribed using the Superscript II kit (Gibco; Thermo Fisher Scientific, Inc.) at $42^{\circ} \mathrm{C}$ for $30 \mathrm{~min}$ followed by enzyme inactivation at $95^{\circ} \mathrm{C}$ for $5 \mathrm{~min}$. The qPCR reactions for $\mathrm{OC}$ were performed using the Rotor-Gene System (Qiagen $\mathrm{GmbH}$ ) and qPCRBIO Probe Mix (Nippon Genetics). Samples were amplified as double replicates by the employment of TaqMan assays specific for OC (Hs01587814_g1 BGLAP) and ACTB (Hs99999903-m1 ACTB). The thermal cycling conditions for TaqMan were as follows: Holding for $10 \mathrm{~min}$ at $95^{\circ} \mathrm{C}, 40$ cycles of $10 \mathrm{sec} / 95^{\circ} \mathrm{C}$ and $30 \mathrm{sec} / 60^{\circ} \mathrm{C}$. OPN and RUNX2 were amplified with 5X HOT FIREPol ${ }^{\circledR}$ EvaGreen $^{\circledR}$ qPCR Mix (Solis Biodyne) using specific primers (Table II) and under following conditions: Holding for $10 \mathrm{~min}$ at $95^{\circ} \mathrm{C}$, followed by 40 cycles of $15 \mathrm{sec}$ at $95^{\circ} \mathrm{C}, 30 \mathrm{sec}$ at $60^{\circ} \mathrm{C}$ and $30 \mathrm{sec}$ at $72^{\circ} \mathrm{C}$. Normalization was performed with primers specific for ACTB, GAPDH, 18S and ACTB TaqMan Probes in the case of OC (Table II). Data evaluation was performed using Rotor-Gene 2.0.2 software and the comparative quantification method $\left(2^{-\Delta \Delta C q}\right)(18)$, which calculated the efficiencies of each gene for each individual PCR reaction (is based on the second differential maximum method (19) to calculate single reaction efficiencies. Briefly, reported concentrations of each PCR-product, including housekeeping genes, were compared to the positive control $(\mathrm{C}+$; cDNA from follicular thyroid cancer cells, FTC-133; cat. no. 94060901-1VL, Sigma-Aldrich; Merck KGaA) set as 1 . Thereafter, all reported concentrations were transformed to percentage values $(\mathrm{C}+$ as $100 \%)$ and target transcripts were normalized to the housekeeping genes. For the whole study, the same $\mathrm{C}+$, the same negative controls and the same housekeeping genes were employed.

$H \& E$ staining. H\&E staining and morphological investigations of selected pathologically changed and not changed vessels were performed. As demonstrated in Fig. 1, freshly cut cryo-embedded serial $6-\mu$ m-thick sections of selected vessel tissues were air-dried at room temperature, shortly washed with cold Acetone (Sigma-Aldrich; Merck KGaA) and dried again. Thereafter, the sections were shortly washed with distilled water and incubated for $1 \mathrm{~min}$ at room temperature in Hämalaun (Merck) solution followed by washing twice with tap water. The sections were then incubated for $1 \mathrm{~min}$ in eosin (Merck KGaA) solution, washed with tap water, shortly washed twice with $96 \%$ ethanol and finally shortly dipped in xylol (Carl Roth GmbH Chemikalien). The sections were then covered with Entellan ${ }^{\circledR}$ (Merck KGaA) and coverslips and examined under a under a light microscope (Axiovert 200; Zeiss GmbH). The staining of BMP4 was performed on cryosections fixed with methanol $/ \mathrm{H}_{2} \mathrm{O}_{2}$ and BMP4 antiserum (Abcam ab155033) diluted 1:100 in TBS. Tissues were lightly counterstained with Mayer's hematoxylin (Merck KGaA) and photographed under a light microscope (Biozero-3000; Keyence).

Immunohistochemistry. Freshly cut cryo-embedded serial $6-\mu \mathrm{m}$-thick sections of selected vessel tissues were washed with PBS and fixed in a 1:4 mixture of $3 \% \mathrm{H}_{2} \mathrm{O}_{2}$ (Carl Roth $\mathrm{GmbH}$ Chemikalien) in ice-cold $90 \%$ methanol (Sigma-Aldrich; Merck KGaA) for 20 min. After washing twice with PBS, the slides were blocked with Envision Flex Antibody Diluent (Dako; Agilent Technologies, Inc.) for $1 \mathrm{~h}$ at room temperature and then incubated overnight at $4^{\circ} \mathrm{C}$ with 
Table I. Clinical data of the patients included in the present study.

\begin{tabular}{|c|c|c|c|c|c|c|c|c|c|c|c|}
\hline $\begin{array}{l}\text { Patient } \\
\text { no. }\end{array}$ & $\begin{array}{l}\text { Age, } \\
\text { years }\end{array}$ & Sex & $\begin{array}{c}\text { Peripheral } \\
\text { artery } \\
\text { disease stage } \\
\text { (Fontaine) }\end{array}$ & $\begin{array}{c}\text { Vessel } \\
\text { location }\end{array}$ & Dyslipidemia & $\begin{array}{c}\text { Diabetes } \\
\text { mellitus } \\
\text { type II }\end{array}$ & $\begin{array}{c}\text { Anti- } \\
\text { vitamin K } \\
\text { anticoagulants }\end{array}$ & $\begin{array}{c}\text { Vitamin D- } \\
\text { supplementation }\end{array}$ & $\begin{array}{l}\mathrm{OC} \\
\text { score }\end{array}$ & $\begin{array}{l}\text { OPN } \\
\text { score }\end{array}$ & $\begin{array}{l}\text { RUNX2 } \\
\text { score }\end{array}$ \\
\hline 1 & 53 & $\mathrm{M}$ & No & Thyroid & No & Yes & No & No & 1 & 2 & 1 \\
\hline 2 & 78 & $\mathrm{M}$ & No & Aorta & No & & No & No & 2 & 2 & 0 \\
\hline 3 & 62 & $\mathrm{M}$ & No & Aorta & - & - & - & - & 1 & 1 & 1 \\
\hline 4 & 78 & $\mathrm{~W}$ & No & Femoral & No & No & No & No & 3 & 2 & 2 \\
\hline 5 & 59 & $\mathrm{~W}$ & No & Neck & No & No & No & No & 2 & 1 & 1 \\
\hline 6 & 65 & $\mathrm{M}$ & No & Thyroid & No & No & No & No & 1 & 1 & 1 \\
\hline 7 & 84 & $\mathrm{M}$ & III & Popliteal & No & No & No & No & 3 & 4 & 4 \\
\hline 8 & 56 & $\mathrm{M}$ & III & Femoral & No & No & No & No & 3 & 6 & 6 \\
\hline 9 & 66 & $\mathrm{M}$ & IV & Femoral & Yes & Yes & No & Yes & 4 & 4 & 9 \\
\hline 10 & 77 & $\mathrm{~W}$ & IV & Femoral & No & Yes & No & No & 4 & 4 & 8 \\
\hline 11 & 97 & $\mathrm{~W}$ & IV & Femoral & Yes & Yes & No & No & 6 & 6 & 4 \\
\hline 12 & 74 & $\mathrm{M}$ & IV & Femoral & No & No & No & No & 4 & 4 & 6 \\
\hline 13 & 83 & $\mathrm{~W}$ & IV & Femoral & Yes & No & No & No & 4 & 6 & 6 \\
\hline
\end{tabular}

OC, OPN and RUNX2 scores determined by multiplying two factors: Overall staining intensity of the slide and specific staining. M, male; F, female.

Table II. Primer pairs for the amplifications of target and housekeeping (HKG) gene transcripts.

\begin{tabular}{llcc}
\hline Gene & \multicolumn{1}{c}{ Primer sequence (5'-3') } & Size (bp) & Supplier \\
\hline RUNX2 & $\begin{array}{l}\text { Forward: CCCTGAACTCTGCACCAAGT } \\
\text { Reverse: GGCTCAGGTAGGAGGGGTAA }\end{array}$ & 120 & Metabion \\
Osteopontin & $\begin{array}{l}\text { Forward: GCCGAGGTGATAGTGTGGTT } \\
\text { Reverse: AACGGGGATGGCCTTGTATG }\end{array}$ & 149 & Metabion \\
HKG & & & Metabion \\
$\beta$-actin & Forward: AGG CAC CAG GGC GTG AT & & Metabion \\
GAPDH & Reverse: GCC CAC ATA GGA ATC CTT CTG AC & 233 & Metabion \\
18 sorward: ACC CAG AAG ACT GTG GAT GG & Reverse: TTC TAG ACG GCA GGT CAG GT & 151 & Thermo Fisher Scientific, Inc. \\
Osteocalcin & Forward: GTT GGT GGA GCG ATT TGT CTG G & & Thermo Fisher Scientific, Inc. \\
HKG & Reverse: AGG GCA GGG ACT TAA TCA ACG C & & \\
\hline
\end{tabular}

the rabbit polyclonal antibody against OC $(2.5 \mu \mathrm{g} / \mathrm{ml} ; \mathrm{ab} 93876$, Abcam), mouse monoclonal antibody against OPN (1:300; ab166709; Abcam) and rabbit polyclonal antibody against RUNX2 (1:1,000; ab23981; Abcam). Negative control sections were exposed to the secondary antibody only and processed as described below. All the following steps were performed at room temperature. After 3x10 min washing in PBS, the slides were incubated for $30 \mathrm{~min}$ at room temperature with a 1:10,00 dilution of biotinylated goat anti-rabbit secondary antibody (sc-2004; Santa Cruz Biotechnology, Inc.) followed by incubation with an avidin-biotin-peroxidase complex (Dako; Agilent Technologies, Inc.). After 3x10 min washing in PBS, specific immunostaining was visualized with diaminobenzidine chromogenic solution (1:50; Dako; Agilent Technologies, Inc.). Finally, the cells were lightly counterstained with Mayer's hematoxylin (Merck KGaA) and photographed under a light microscope (Axiovert 200; Zeiss GmbH) (Fig. 1).

Two independent reviewers, semi-quantitatively using an Axioplan light microscope (Zeiss $\mathrm{GmbH}$ ), examined all immunostained tissue sections. The OC, OPN and RUNX2 scores was determined by multiplying two factors: The oerall staining intensity of the slide and specific staining. In overall staining, the slides were evaluated according to a 0-3 points scale, where 0 indicates negative staining and 3 the strongest 
A

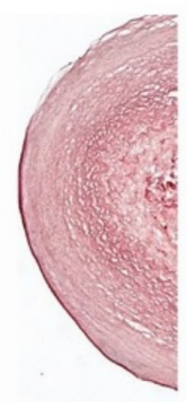

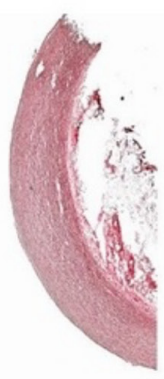

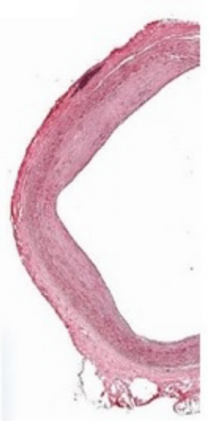

B

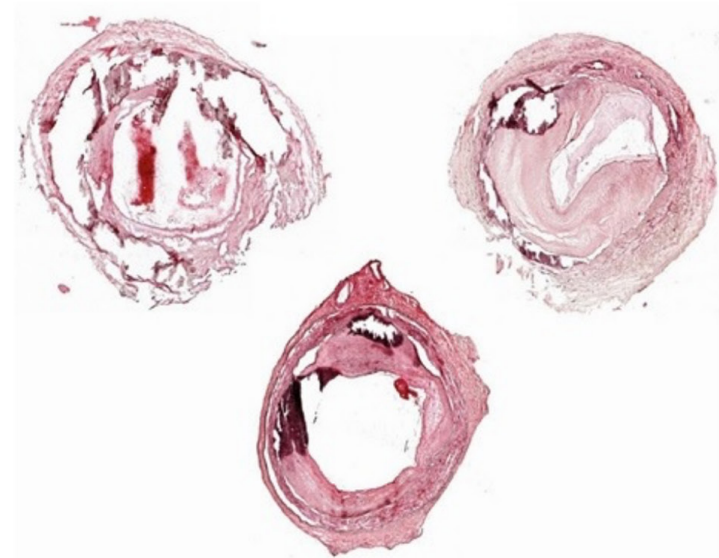

Figure 1. Representative H\&E staining of (A) healthy and (B) diseased vessels obtained from PAD- and PAD+ femoral arteries. (A) Normal-appearing femoral artery with no evidence of intimal thickening or medial calcification and (B) adaptive intimal thickening with focal calcification of the internal elastic lamina. Magnification: Healthy vessels, x5; diseased vessels, x2.5. There are three representative images of PAD- and PAD+ shown for each image. H\&E, hematoxylin and eosin; PAD-, patients without arteriosclerotic disease; PAD+ patients with arteriosclerotic disease.

staining. The points for specific staining were calculated as follows: $0,0-10 \%$ (negative); 1, 10-30\% (low expression); 2, 30-50\% (moderate expression); 3, 50-80\% (high expression); $4, \geq 80 \%$ (very high expression).

Statistical analysis. Each experiment was performed in quadruplicate. The distribution of the quantitative variables within qPCR data of all investigated transcripts was tested with Kolmogorov-Smirnov normality tests. Since the expression of OC, OPN and RUNX2 passed the normality tests, a parametric (differences between paired values are consistent) two-sided t-test was used. Data are presented as medians with inter quartile ranges. For comparisons within vascular tissues, the Bonferroni correction was applied and $\mathrm{P}$-values $\mathrm{P}<0.01$, $\mathrm{P}<0.001$ and $\mathrm{P}<0.0001$ were considered to indicated statistically significant differences. All analyses were performed using GraphPad Prism 6 software (GraphPad Software, Inc.). Data are presented as the median and inter quartile range.

\section{Results}

Evidence of calcification in diseased tissues. H\&E staining revealed that tissues from PAD- appeared normal and exhibited no evidence of intimal thickening or medial calcification (Fig. 1A). However, the femoral artery tissues from PAD+ exhibited adaptive intimal thickening with focal calcification of the internal elastic lamina (Fig. 1B).

Expression and immunostaining of $O C$. The analysis of the transcript levels revealed that the mRNA expression of OC was markedly higher in PAD+ tissues than in corresponding PADtissues (Fig. 2A). The median percentage transcript expression for OC in PAD+ was $634 \%$ and $88.5 \%$ in PAD- $(\mathrm{P}=0.067)$. Specific staining with anti-OC antibody demonstrated the same tendency as the transcript analyses. As shown in Fig. 2A, a more intense $\mathrm{OC}$ expression was detected in the PAD+ as compared with the PAD- tissues.

Expression and immunostaining of $O P N$. As regards the mRNA expression of OPN, this was significantly elevated in
PAD+ tissues compared with the PAD- group (Fig. 2B). The median percentage transcript expression for OPN in $\mathrm{PAD}+$ was $1,015 \%$ and $322.5 \%$ for PAD- $(\mathrm{P}=0.0085)$. Specific staining with anti-OPN antibody also demonstrated the same tendency as the transcript analyses (Fig. 2B).

Expression and immunostaining of RUNX2. A significantly elevated mRNA expression of RUNX2 was observed in the PAD+ group compared with the PAD- group (Fig. 2C). The median percentage transcript expression for RUNX2 in PAD+ was $117 \%$ and $64 \%$ in PAD- $(\mathrm{P}=0.0134)$. As shown in Fig. 2C, a more intense OC expression was also detected in PAD+ as compared with PAD-.

Representative staining of BMP4 in healthy and diseased vessels. Representative staining for BMP4 in healthy (Fig. 3A) and diseased vessels (Fig. 3B) was also performed. Vessels obtained from arteriosclerotic patients exhibited much more intense staining for BMP4 than those obtained from healthy subjects.

\section{Discussion}

In the present study, human vessel tissues originating from patients with or without arteriosclerotic disease were investigated. One key finding was that the expression of OC, OPN and RUNX2 in arterial tissue was strongly associated with arteriosclerotic disease. This indicated that these factors have a valuable diagnostic potential. However, the biological role of OC, OPN and RUNX2 in arteriosclerotic disease is not yet fully understood.

$O C$. OC has been described as a potential preventive or therapeutic agent in metabolic disorders (20). The complex expression of OC suggests that all its functions are not yet known (21). A recent meta-analysis revealed an overall significant inverse association between serum OC levels and body mass index (22). In mouse models and patients with peripheral artery disease, circulating OC-positive mononuclear cells have been found to be associated with severe calcification of the aorta (23). 
A
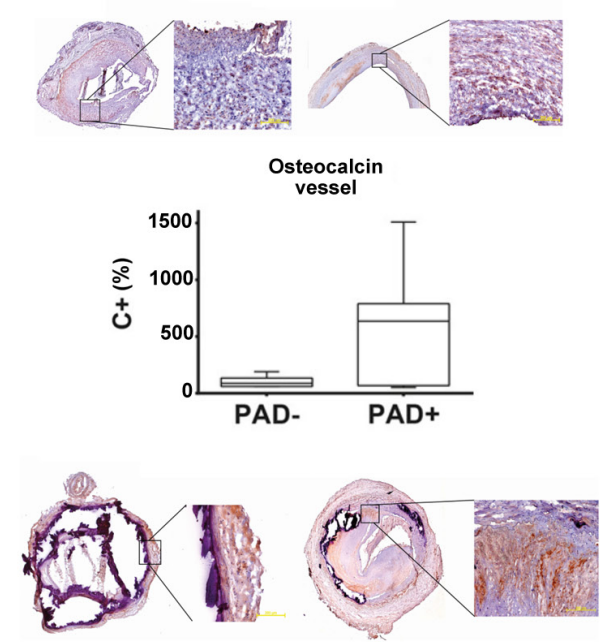

B
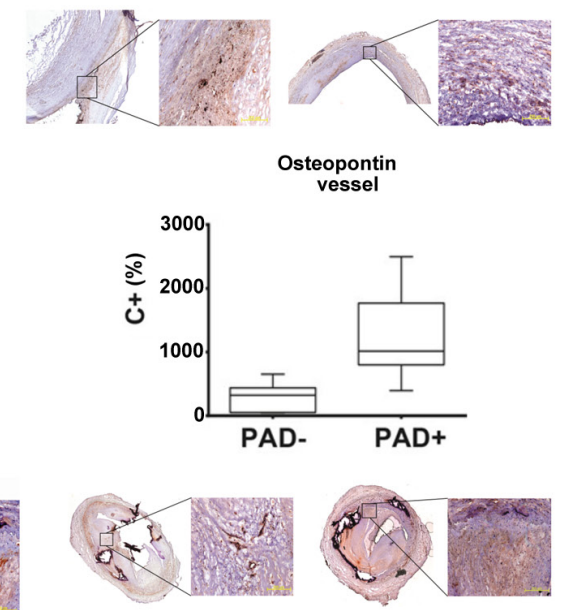

C
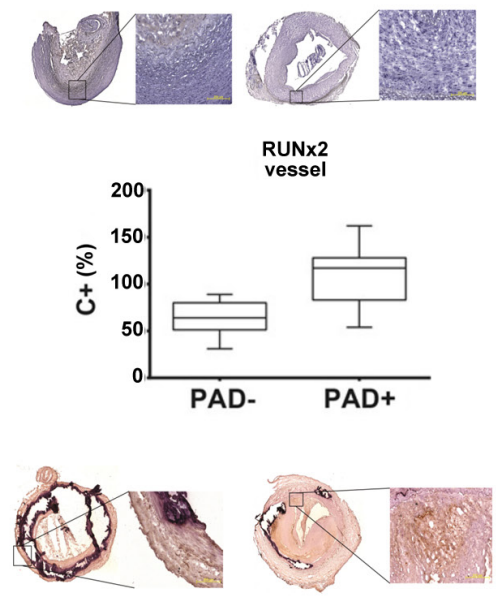

Figure 2. Analysis of the mRNA expression of (A) OC, (B) OPN and (C) RUNX2 in vascular tissue from PAD+ (top panels) and PAD- (bottom panels). An elevated mRNA expression of OC, OPN and RUNX2 was found in the PAD+ group (middle panels). The median percentage transcript expression for OC in $\mathrm{PAD}+$ was $634 \%$ and $88.5 \%$ for PAD- $(\mathrm{P}=0.067)$. The median percentage transcript expression for OPN in $\mathrm{PAD}+\mathrm{was} 1015 \%$ and $322.5 \%$ for $\mathrm{PAD}-(\mathrm{P}=0.0085)$. The median percentage transcript expression for RUNX2 in PAD+ was $117 \%$ and for $64 \%$ PAD- $(\mathrm{P}=0.0134)$. The expression of all transcripts was compared to the positive control (C+) set as $100 \%$ and presented as \% values as compared to $\mathrm{C}+$. (A-C) There are two images of PAD- tissues (top panels) and two images of PAD+ tissues shown (bottom panels) for each marker. The images in all panels are presented at magnifications of x 2.5 and zoomed in at $x 10$, respectively.

\section{BMP4}

Healthy

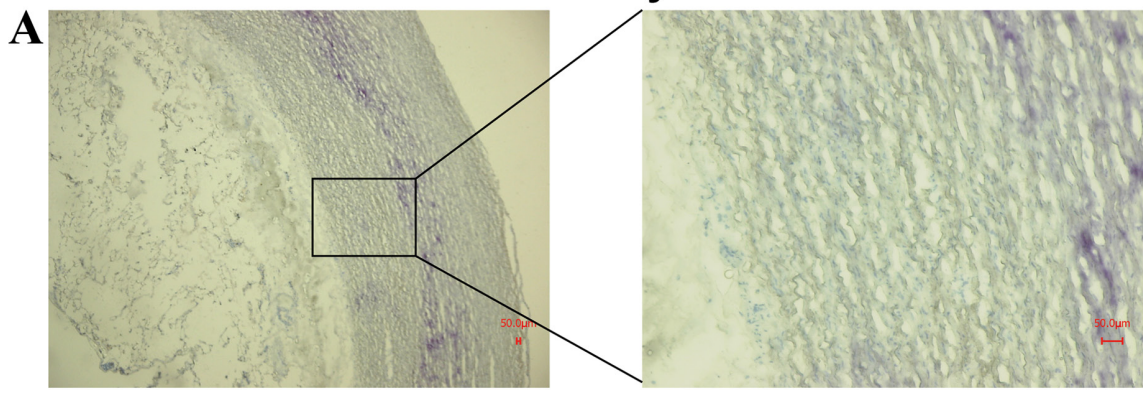

Diseased

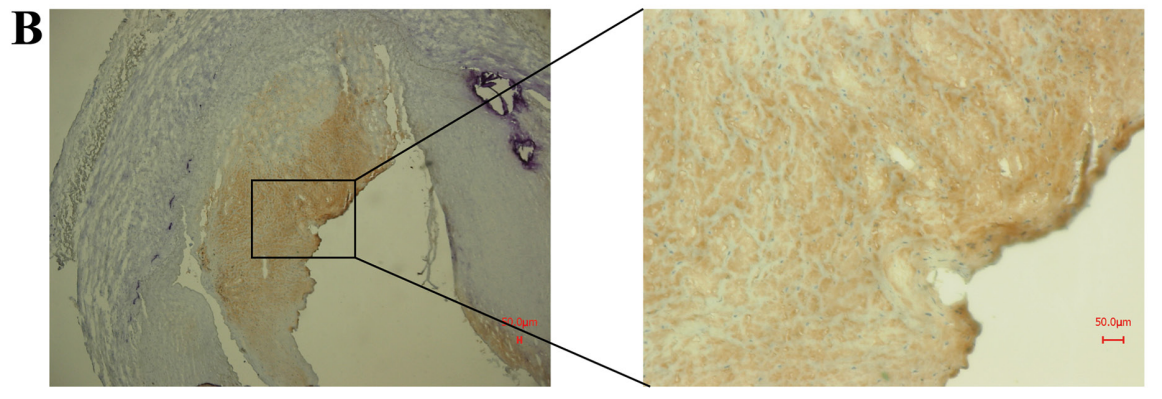

Figure 3. Representative staining of BMP4 in (A) healthy and (B) diseased vessels. The staining was performed on cryosections fixed with methanol/ $\mathrm{H}_{2} \mathrm{O}_{2}$ and BMP4 antiserum Note that vessels obtained from arteriosclerotic patients demonstrated much more intense staining for BMP4 than healthy vessels.

In the present study, a clear association was found between the levels of OC expression and symptomatic arteriosclerotic disease. In a previous study, the same association was made. Yuen et al (24) investigated the molecular mechanisms underlying the promoting effects of $\mathrm{OC}$ on vascular pathogenesis and remodeling. The main finding of that study was that $\mathrm{OC}$ transforms adventitial fibroblasts into myofibroblasts by stimulating angiotensin II release and the subsequent activation of the protein kinase $\mathrm{C} \delta(\mathrm{PKC}) / \mathrm{Toll}$ like receptor 4 (TLR4)/reactive oxygen species/cyclooxygenase-2 signaling cascade (24). On the other hand, in another study, no significant association between vascular calcification and OC (25) was found in patients undergoing peritoneal dialysis.

$O P N$. In a recent study from Italy, OPN plasma levels were found to be associated with type II diabetes and obesity (26). 
OPN is also referred to as a marker of the outcome of patients with vascular diseases, including peripheral artery disease $(13,27)$. In mice, OPN has been found to be an inducible inhibitor of vascular calcification (28). In adidtion, the plasma levels of OPN have been shown to be associated with cardiovascular pathologies, such as abdominal aortic aneurysm (29,30). Furthermore, elevated plasma levels of OPN have been discussed as an independent predictor of coronary calcification in patients with diabetes and asymptomatic coronary disease (31). In the present study, OPN was found to be strongly associated with symptomatic arteriosclerotic disease. The findings presented herein are consistent with previous findings that OPN may be a marker of the disease (32).

RUNX2. Recently, RUNX2 activity and arteriosclerotic vascular calcification were found to be strongly associated in patients with diabetes mellitus (33). In another study, Tanaka et al (34) stated that RUNX2 played a major role in osteogenic conversion in arteriosclerotic lesions. In mouse models, RUNX2 has been shown to be strongly associated with the vessel calcification process $(35,36)$. These findings support the results of the present study and identify RUNX2 as a novel potential drug target in the treatment of patients with severe calcification due to arteriosclerosis.

It was hypothesized that the diseased vessel wall may act as chemoattractant for immune cells, which in turn produce increased amounts of cytokines and affect endothelial and muscle cells. These in turn react with increased or modulated expression of OC, OPN and RUNX2. In addition, the immune cells infiltrate vessel wall and induce signaling pathways affecting OC, OPN and RUNX2. Finally, the increased calcium and/or phosphate levels lead to the dysfunction of immune cells and/or endothelial cells/muscle cells. As a result, the levels of OC, OPN and RUNX2 are increased.

The present study has some limitations. The present study lacks functional data, particularly as regards the influence of $\mathrm{OC}$, OPN and RUNX2-modifying medication on the progression of arteriosclerotic disease. In addition, whether circulating blood levels of OC, OPN and RUNX2 in serum/plasma by means of a non-invasive assay may support the development of the disease. Furthermore, the differences in the investigated vessel types for PAD- compared with PAD+ (all femoral) may represent a limitation of the present study. The present pilot study was also conducted on a small patient collective. However, on the whole, the promising results presented herein justify future investigations using larger cohorts and make possible the more effective diagnosis and follow-up of these patients.

In conclusion, in the present study, the expression of $\mathrm{OC}$, OPN and RUNX2 in arterial tissue was found to be strongly associated with symptomatic arteriosclerotic disease. However, the role that these three markers play in the development of CVD remains unclear. Further investigations are required to evaluate the roles of OC, OPN and RUNX2 as potential biomarkers in the diagnosis and even the follow-up of these patients.

\section{Acknowledgements}

The authors would like to thank Ms. Kathrin Hammje from Martin Luther University Halle-Wittenberg for providing excellent technical assistance with the creation of this manuscript.

\section{Funding}

No funding was received.

\section{Availability of data and materials}

The datasets used and/or analyzed during the current study are available from the corresponding author on reasonable request.

\section{Authors' contributions}

JU and BT were involved in the conceptualization of the study. BT was involved in the study methodology and in the provision of software. CHV was involved in data validation. BT and $\mathrm{CHV}$ were involved in formal analysis. JU and AR were involved in the investigative aspects of the study, as well as in data curation, study supervision and project administration. AR was involved in the provision of resources, in the writing of the original draft, in the reviewing and editing of the manuscript. All authors confirm the authenticity of all the raw data and all authors have read and approved the final manuscript.

\section{Ethics approval and consent to participate}

The Ethics Committee of the Martin Luther University, Faculty of Medicine, approved the study and all patients provided written informed consent.

\section{Patient consent for publication}

Not applicable.

\section{Competing interests}

The authors declare that they have no competing interests.

\section{References}

1. Ferron M and Lacombe J: Regulation of energy metabolism by the skeleton: Osteocalcin and beyond. Arch Biochem Biophys 561: 137-146, 2014.

2. Fishbein GA and Fishbein MC: Arteriosclerosis: Rethinking the current classification. Arch Pathol Lab Med 133: 1309-1316, 2009.

3. Rennenberg RJ, Kessels AG, Schurgers LJ, van Engelshoven JM, de Leeuw PW and Kroon AA: Vascular calcifications as a marker of increased cardiovascular risk: A meta-analysis. Vasc Health Risk Manag 5: 185-197, 2009.

4. Shobeiri N, Adams MA and Holden RM: Vascular calcification in animal models of CKD: A review. Am J Nephrol 31: 471-481, 2010.

5. World Health Organization: Cardiovascular Diseases. https:// www.who.int/health-topics/cardiovascular-diseases/\#tab=tab_1. Accessed December 2, 2020.

6. Wexler L, Brundage B, Crouse J, Detrano R, Fuster V, Maddahi J, Rumberger J, Stanford W, White R and Taubert K: Coronary artery calcification: Pathophysiology, epidemiology, imaging methods, and clinical applications. Circulation 94: 1175-1192, 1996.

7. Zoch ML, Clemens TL and Riddle RC: New insights into the biology of osteocalcin. Bone 82: 42-49, 2016.

8. Lee NK, Sowa H, Hinoi E, Ferron M, Ahn JD, Confavreux C, Dacquin R, Mee PJ, McKee MD, Jung DY, et al: Endocrine regulation of energy metabolism by the skeleton. Cell 130: 456-469, 2007.

9. Sheng L, Cao W, Cha B, Chen Z, Wang F and Liu J: Serum osteocalcin level and its association with carotid atherosclerosis in patients with type 2 diabetes. Cardiovasc Diabetol 12: 22, 2013. 
10. Tacey A, Qaradakhi T, Brennan-Speranza T, Hayes A, Zulli A and Levinger I: Potential role for osteocalcin in the development of atherosclerosis and blood vessel disease. Nutrients 10: E1426, 2018.

11. Millar SA, Patel H, Anderson SI, England TJ and O'Sullivan SE: Osteocalcin, vascularcalcification, and atherosclerosis: A systematic review and meta-analysis. Front Endocrinol (Lausanne) 8: 183, 2017.

12. Lund SA, Giachelli CM and Scatena M: The role of osteopontin in inflammatory processes. J Cell Commun Signal 3: 311-322, 2009.

13. Lok ZSY and Lyle AN: Osteopontin in vascular disease. Arterioscler Thromb Vasc Biol 39: 613-622, 2019.

14. Moe SM, Duan D, Doehle BP, O'Neill KD and Chen NX: Uremia induces the osteoblast differentiation factor Cbfa1 in human blood vessels. Kidney Int 63: 1003-1011, 2003.

15. Tyson KL, Reynolds JL, McNair R, Zhang Q, Weissberg PL and Shanahan CM: Osteo/chondrocytic transcription factors and their target genes exhibit distinct patterns of expression in human arterial calcification. Arterioscler Thromb Vasc Biol 23: 489-494, 2003.

16. Li Y, Wang W, Chao Y, Zhang F and Wang C: CTRP13 attenuates vascular calcification by regulating Runx2. FASEB J 33: 9627-9637, 2019.

17. Zhu L, Zhang N, Yan R, Yang W, Cong G, Yan N, Ma W, Hou J, Yang L and Jia S: Hyperhomocysteinemia induces vascular calcification by activating the transcription factor RUNX2 via Krüppel-like factor 4 up-regulation in mice. J Biol Chem 294 19465-19474, 2019.

18. Livak KJ and Schmittgen TD: Analysis of relative gene expression data using real-time quantitative PCR and the $2(-\Delta \Delta \mathrm{C}(\mathrm{T}))$ method. Methods 25: 402-408, 2001.

19. Rasmussen R: Quantification on the Light Cycler. In: Rapid Cycle Real-Time PCR. Meuer S, Wittwer C and Nakagawara KI (eds). Springer, Heidelberg, pp21-34 2001.

20. Mizokami A, Kawakubo-Yasukochi T and Hirata M: Osteocalcin and its endocrine functions. Biochem Pharmacol 132: 1-8, 2017.

21. Li J, Zhang H, Yang C, Li Y and Dai Z: An overview of osteocalcin progress. J Bone Miner Metab 34: 367-379, 2016.

22. Kord-Varkaneh H, Djafarian K, Khorshidi M and Shab-Bidar S: Association between serum osteocalcin and body mass index: A systematic review and meta-analysis. Endocrine 58: 24-32, 2017.

23. Pal SN, Rush C, Parr A, Van Campenhout A and Golledge J: Osteocalcin positive mononuclear cells are associated with the severity of aortic calcification. Atherosclerosis 210: 88-93, 2010

24. Yuen CY, Wong SL, Lau CW, Tsang SY, Xu A, Zhu Z, Ng CF, Yao X, Kong SK, Lee HK, et al: From skeleton to cytoskeleton: Osteocalcin transforms vascular fibroblasts to myofibroblasts via angiotensin II and Toll-like receptor 4. Circ Res 111: e55-e66, 2012.

25. Ramirez-Sandoval JC, Casanova I, Villar A, Gomez FE, Cruz C and Correa-Rotter R: Biomarkers associated with vascular calcification in peritoneal dialysis. Perit Dial Int 36: 262-268, 2016.
26. Schinzari F, Tesauro M, Bertoli A, Valentini A, Veneziani A, Campia U and Cardillo C: Calcification biomarkers and vascular dysfunction in obesity and type 2 diabetes: Influence of oral hypoglycemic agents. Am J Physiol Endocrinol Metab 317: E658-E666, 2019.

27. Icer MA and Gezmen-Karadag M: The multiple functions and mechanisms of osteopontin. Clin Biochem 59: 17-24, 2018.

28. Speer MY, McKee MD, Guldberg RE, Liaw L, Yang HY, Tung E, Karsenty G and Giachelli CM: Inactivation of the osteopontin gene enhances vascular calcification of matrix Gla protein-deficient mice: Evidence for osteopontin as an inducible inhibitor of vascular calcification in vivo. J Exp Med 196: 1047-1055, 2002

29. Scatena M, Liaw L and Giachelli CM: Osteopontin: A multifunctional molecule regulating chronic inflammation and vascular disease. Arterioscler Thromb Vasc Biol 27: 2302-2309, 2007.

30. Golledge J, Muller J, Shephard N, Clancy P, Smallwood L, Moran C, Dear AE, Palmer LJ and Norman PE: Association between osteopontin and human abdominal aortic aneurysm. Arterioscler Thromb Vasc Biol 27: 655-660, 2007.

31. Berezin AE and Kremzer AA: Circulating osteopontin as a marker of early coronary vascular calcification in type two diabetes mellitus patients with known asymptomatic coronary artery disease. Atherosclerosis 229: 475-481, 2013.

32. Cho HJ, Cho HJ and Kim HS: Osteopontin: A multifunctional protein at the crossroads of inflammation, atherosclerosis, and vascular calcification. Curr Atheroscler Rep 11: 206-213, 2009.

33. Lino M, Wan MH, Rocca AS, Ngai D, Shobeiri N, Hou G, Ge C, Franceschi RT and Bendeck MP: Diabetic vascular calcification mediated by the collagen receptor discoidin domain receptor 1 via the phosphoinositide 3-kinase/Akt/Runt-related transcription factor 2 signaling axis. Arterioscler Thromb Vasc Biol 38: 1878-1889, 2018

34. Tanaka T, Sato H, Doi H, Yoshida CA, Shimizu T, Matsui H, Yamazaki M, Akiyama H, Kawai-Kowase K, Iso T, et al: Runx2 represses myocardin-mediated differentiation and facilitates osteogenic conversion of vascular smooth muscle cells. Mol Cell Biol 28: 1147-1160, 2008.

35. Al-Huseini I, Ashida N and Kimura T: Deletion of IкB-Kinase $\beta$ in smooth muscle cells induces vascular calcification through $\beta$-catenin-Runt-related transcription factor 2 signaling. J Am Heart Assoc 7: e007405, 2018.

36. Deng L, Huang L, Sun Y, Heath JM, Wu H and Chen Y: Inhibition of FOXO1/3 promotes vascular calcification. Arterioscler Thromb Vasc Biol 35: 175-183, 2015.

(i) $($ This work is licensed under a Creative Commons Attribution-NonCommercial-NoDerivatives 4.0 International (CC BY-NC-ND 4.0) License. 